\title{
Type 3 peptide deformylases are required for oxidative phosphorylation in Trypanosoma brucei
}

\author{
Nabile Bouzaidi-Tiali, ${ }^{1}$ Carmela Giglione, ${ }^{2}$ \\ Yannick Bulliard, ${ }^{1 \dagger}$ Mascha Pusnik, ${ }^{1}$ Thierry Meinnel ${ }^{2}$ \\ and André Schneider ${ }^{1 *}$ \\ ${ }^{1}$ Department of Biology/Cell and Developmental Biology, \\ University of Fribourg, Chemin du Musée 10, CH-1700 \\ Fribourg, Switzerland. \\ ${ }^{2}$ Institut des Sciences du Végétal, UPR2355 Centre \\ National de la Recherche Scientifique, CNRS, Bâtiment \\ 23, Avenue de la Terrasse, F-91198 Gif-sur-Yvette \\ cedex, France.
}

\section{Summary}

Peptide deformylase (PDF) catalyses the removal of the formyl group from the first methionine of nascent proteins. Type 1 PDFs are found in bacteria and have orthologues in most eukaryotes. Type 2 PDFs are restricted to bacteria. Type 3 enzymes are found in Archaea and trypanosomatids and have not been studied experimentally yet. Thus, TbPDF1 and TbPDF2, the two PDF orthologues of the parasitic protozoa Trypanosoma brucei, are of type 3. An experimental analysis of these enzymes shows that both are mitochondrially localized, but that only TbPDF1 is essential for normal growth. Recombinant TbPDF1 exhibits PDF activity with a substrate specificity identical to that of bacterial enzymes. Consistent with these results, TbPDF1 is required for oxidative but not for mitochondrial substrate-level phosphorylation. Ablation of TbPDF2, in contrast, does neither affect growth on standard medium nor oxidative phosphorylation. However, a reduced level of TbPDF2 slows down growth in a medium that selects for highly efficient oxidative phosphorylation. Furthermore, combined ablation of TbPDF1 and TbPDF2 results in an earlier growth arrest than is observed by downregulation of TbPDF1 alone. These results suggest that TbPDF2 is functionally linked to TbPDF1, and that it can influence the efficiency of oxidative phosphorylation.

${ }^{*}$ For correspondence. E-mail andre. schneider@unifr.ch; Tel. (+41) 263008877; Fax (+41) 263009741; E-mail Thierry.Meinnel@isv.cnrs-gif.fr; Tel. (+33) 169823612; Fax (+33) 169823607. 'Present address: School of Life Sciences, Ecole Polytechnique Fédérale de Lausanne (EPFL), CH-1015 Lausanne, Switzerland.

\section{Introduction}

Bacterial and organellar initiator tRNAs ${ }^{\mathrm{Met}}$ are only functional when the methionine they carry is formylated. Addition of the formyl group to the charged initiator tRNAMet is catalysed by the methionyl-tRNA ${ }^{\text {Met }}$ formyltransferase (MTF). During translation initiation, the formylated initiator tRNA ${ }^{\text {Met }}$ interacts with the bacterial-type initiation factor 2 , and the resulting complex binds to the ribosome in a GTP-dependent way (Mayer et al., 2001). Thus, newly synthesized proteins emerging from ribosomes of bacteria and organelles carry formyl-methionines at their $\mathrm{N}$-termini. In many cases, this formyl-methionine needs to be removed for the protein to be functional (Giglione and Meinnel, 2001a; Giglione et al., 2004). However, aminopeptidases, which cleave off $\mathrm{N}$-terminal methionines, can only process methionines that are not formylated. Therefore, a peptide deformylase (PDF) is needed which removes the formyl group (Giglione and Meinnel, 2001a; Giglione et al., 2004).

For many years it was thought that PDFs are specific for bacteria, and they were therefore considered to be excellent targets for antibacterial drugs. Indeed, several PDF inhibitors are being tested in clinical trials (Boularot et al., 2004; Leeds and Dean, 2006). However, more recent studies have shown that PDF homologues are also encoded in essentially all eukaryotic and in some archaeal genomes. The few eukaryotic PDFs that have been studied in more detail are essential proteins (Giglione et al., 2003; Lee et al., 2004) that are targeted to chloroplasts and to mitochondria (Lee et al., 2004; Nguyen et al., 2003; Serero et al., 2003).

Trypanosoma brucei is a parasitic protozoa that occurs in sub-Saharan Africa. It has a complex life cycle and is transmitted between mammalian hosts through a tsetse fly vector. T. brucei causes human sleeping sickness and various diseases in other animals. If left untreated, sleeping sickness is invariable fatal. However, treatment of the disease is still in a very unsatisfactory state, and the only effective drug against the late stage of the disease, which is in use for more than 50 years, causes death in 3-10\% of the patients (Lüscher et al., 2007). T. brucei is related to Trypanosoma cruzi, the causative agent of Chagas disease in Latin America, as well as Leishmania spp. that occur in much of the tropical and subtropical world and that cause the different forms of leishmaniasis. 
As in other eukaryotes, mitochondrial translation in the parasitic protozoa $T$. brucei is of the bacterial-type (Schneider, 2001). Consistent with this, it was shown that mitochondrial translation initiation in $T$. brucei requires a formylated tRNA ${ }^{\text {Met }}$ (Tan et al., 2002). However, due to the complete lack of mitochondrial tRNA genes (Schneider and Marechal-Drouard, 2000; Bhattacharyya and Adhya, 2004), it is not a bacterial-type initiator tRNA ${ }^{\text {Met }}$ that becomes formylated, but the elongator tRNA ${ }^{\text {Met }}$ that is imported from the cytosol. The formylation is catalysed by an MTF that, unlike its counterparts in other organisms, recognizes the imported elongator tRNA ${ }^{\text {Met }}$. Thus, we find the surprising situation in $T$. brucei mitochondria that the elongator tRNA ${ }^{\text {Met }}$ is functional in translation initiation provided that it is formylated (Martin, 2002). This highlights how crucial tRNA formylation is in mitochondria. In agreement with these findings, it was shown that the main determinant recognized by the mitochondrial initiation factor 2 of $T$. brucei is the formylated methionine (Charrière et al., 2005). It is therefore clear that newly synthesized mitochondrially encoded proteins of $T$. brucei carry an $\mathrm{N}$-terminal formyl-methionine.

Mitochondrial translation in T. brucei has proven to be difficult to study. Thus, except for two mitochondrially synthesized proteins that are probably not processed, it is not known whether the formyl group is removed (Horvath et al., 2000a, b). Here we show that the T. brucei genome encodes two distinct putative PDF orthologues. One of the proteins was recombinantly expressed and exhibited deformylase activity. Furthermore, as predicted for deformylases, both T. brucei PDF orthologues are localized in mitochondria and required for efficient oxidative phosphorylation (OXPHOS), a process that in part depends on mitochondrially encoded proteins.

\section{Results}

Two PDF orthologues in T. brucei

The $T$. brucei genome encodes open reading frames (ORFs) for two putative PDF orthologues. The proteins have a predicted molecular weight of $30 \mathrm{kDa}$ (TbPDF1: accession numbers Tb11.01.6120 and AF177768] and 42 kDa (TbPDF2: accession number Tb09.211.0190] respectively. They contain the three signature sequences that define the PDF protein family (Giglione and Meinnel, 2001b; Meinnel, 2000), even though motif 1 lacks the conserved glutamine residue that appears to be crucial for the recognition of the formyl group by TbPDF1 (Meinnel et al., 1997) (Fig. 1). The two T. brucei PDFs share only a limited identity of $30 \%$ that is restricted to the C-terminal half of TbPDF1. However, both proteins have conserved orthologues in Leishmania major and T. cruzi with which they share an overall identity of $63 \%$ (for TbPDF1) and
$75 \%$ (for TbPDF2). Interestingly, unlike the TbPDF2, the first PDF signature sequence of the L. major and the T. cruzi TbPDF2 orthologues includes the conserved glutamine residue of motif 1 (Fig. 1). The closest nontrypanosomatidal orthologues that are identified by BLAST analysis are the PDF1B of Arabidopsis thaliana (for TbPDF1) and the PDF of the bacteria Leptospira interrogans (for TbPDF2). Finally, compared with bacterial PDFs, trypanosomatidal PDF orthologues have both an $\mathrm{N}$ - and a C-terminal extension.

\section{Biochemical analysis of recombinant TbPDF1}

Based on changes in the conserved motifs, it has been suggested in a recent phylogenetic analysis that trypanosomal and archaeal PDFs form a separate group, termed type 3 PDFs (Giglione et al., 2004). Thus, in order to test whether the trypanosomal PDF orthologues have PDF activity, we prepared recombinant TbPDF1, the more conserved of the two trypanosomal PDFs. To do so, fulllength TbPDF1 and three variants thereof lacking either the first 30 amino acids (TbPDF1/31-260), the last 51 amino acids (TbPDF1/1-209) or both (TbPDF1/31-209) were expressed in Escherichia coli (Fig. 2A). All proteins carried a hexahistidine tag at their $\mathrm{C}$-termini. Analysis of crude extracts with anti-hexahistidine tag antibodies showed that, whereas TbPDF1/31-260 was expressed at high levels, only low amounts of the other TbPDF1 variants accumulated (not shown). Thus, the presence of the $\mathrm{N}$-terminal 30 amino acids and the absence of the C-terminal 51 amino acids had a deleterious effect on TbPDF1 expression.

Using nickel column affinity chromatography, the highly expressed TbPDF1/31-260 variant was purified to homogeneity as illustrated by the Coomassie-stained gel in Fig. 2B. By the same method, we obtained highly enriched fraction for full-length TbPDF1 and the TbPDF1/ 31-209 variant. Expression of the TbPDF1/1-209 variant was very weak. The corresponding nickel column eluate was poorly enriched in recombinant protein as a result, and contained a major contaminant that co-migrates with the recombinant protein as evidenced by immunoblot analysis using anti-Tag antibodies (Fig. 2B, lane TbPDF1/ 1-209).

Previous work had indicated that the catalytic core of bacterial PDFs is highly resistant to trypsin digestion (Meinnel et al., 1996). Thus, we expect TbPDF1 to behave the same way. The predicted catalytic core of TbPDF1, encompassing approximately residues 36-198, is present in all four recombinant TbPDF1 variants. Consistent with this, low concentrations of trypsin were sufficient to convert full-length TbPDF1 into a trypsin-resistant fragment of approximately $28 \mathrm{kDa}$ (Fig. $2 \mathrm{C}$, top panel). The fragment still reacted with the anti-His-tag antibodies, 


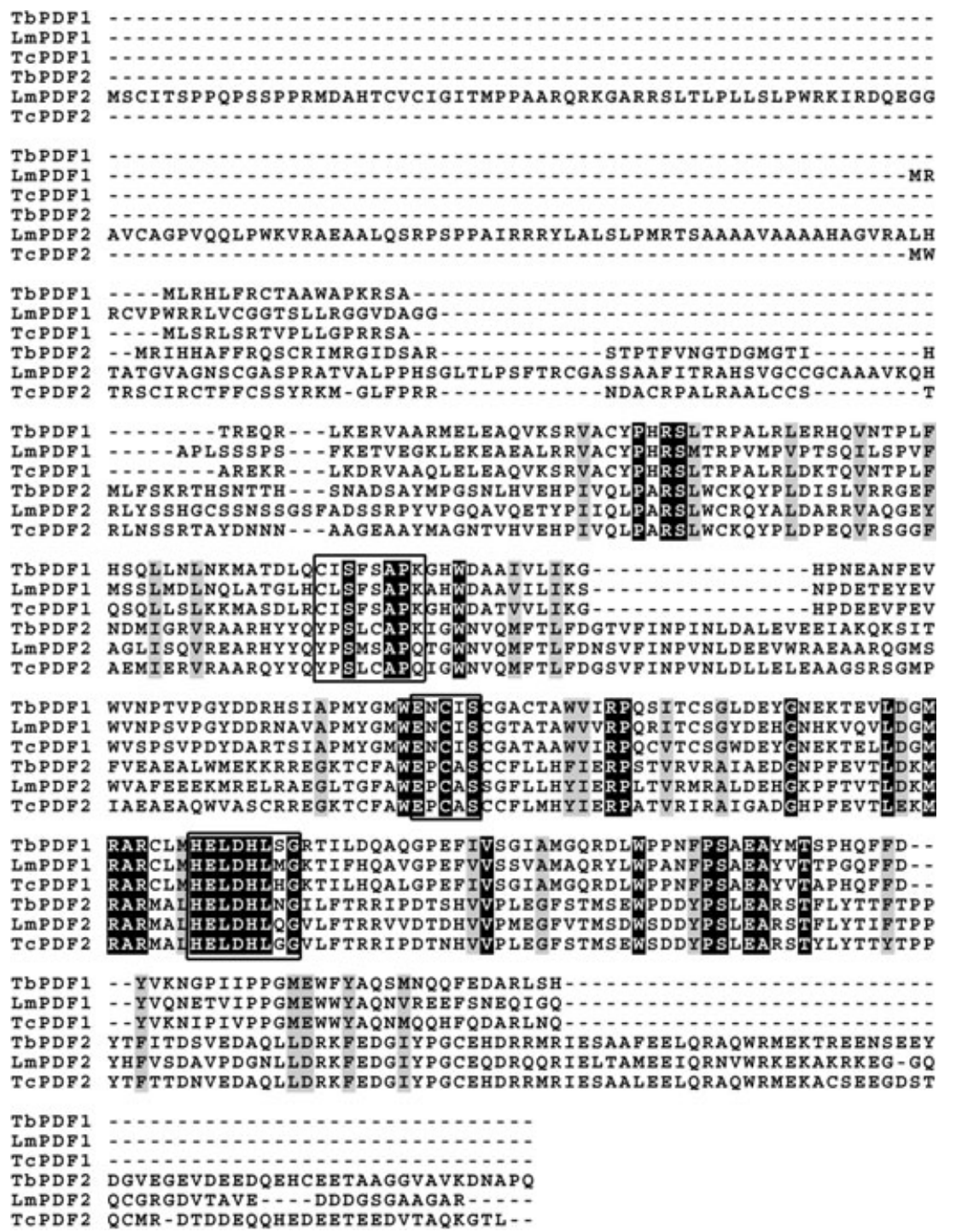

Fig. 1. Trypanosoma brucei contains two PDF orthologues. Multiple sequence alignment of the TbPDF1 and TbPDF2 and their corresponding orthologues in Leishmania major (LmPDF1, LmPDF2) and in

Trypanosoma cruzi (TcPDF1, TcPDF2). The sequences were aligned using the CLUSTAL $W$ program with default parameters. Strictly conserved residues and conservative replacements are shown in black and grey boxes respectively. The three PDF signature sequences (Giglione and Meinnel, 2001b) are indicated in the three large boxes. indicating that its C-terminus was intact. TbPDF1/31-260 co-migrated with the proteolytic fragment of the full-length enzyme and resisted proteolytic attack within the same range of concentration (Fig. $2 \mathrm{C}$, middle panel). In contrast, TbPDF1/31-209 (Fig. 2C, bottom panel) and TbPDF1/1-209 (not shown) were completely hydrolysed at very low concentrations of trypsin. These experiments show that TbPDF1 has a protease-resistant core similar in size to the one found in bacterial PDFs. Furthermore, the removal of the N-terminus at very low trypsin concentrations suggests that, in agreement with previous analyses of PDFs from other organisms, this extension has no defined secondary structure.

Finally, full-length recombinant TbPDF1 and the most stable truncated variant TbPDF1/31-260 were assayed for deformylation activity. Both proteins were able to remove the formyl group from formylated peptides consisting of two or more amino acids in length (Table 1).
Highest activities were observed for dipeptides containing either formylated methionine or phenylalanine. Interestingly, however, a dipeptide containing isoleucine, even though it is also hydrophobic in nature, was a much worse substrate (Table 1). Thus, the substrate specificity of TbPDF1 is identical to that of bacterial PDFs, which also discriminate between phenylalanine and isoleucine (Ragusa et al., 1999).

The observed specific activity of TbPDF1 was very low $\left(\mathrm{k}_{\text {cat }} / \mathrm{K}_{\mathrm{M}}=8 \mathrm{M}^{-1} \mathrm{~s}^{-1}\right)$ but similar to the one observed for human (Serero et al., 2001; Serero et al., 2003; Lee et al., 2004) and Plasmodium falciparum PDFs (Bracchi-Ricard et al., 2001) when tested with comparable substrates. Specific activities of the same order of magnitude have also been described for some bacterial PDFs. In these cases, the low activities could be explained by an exchange of the metal cation found in the active site in vivo for another less efficient one that may render the 


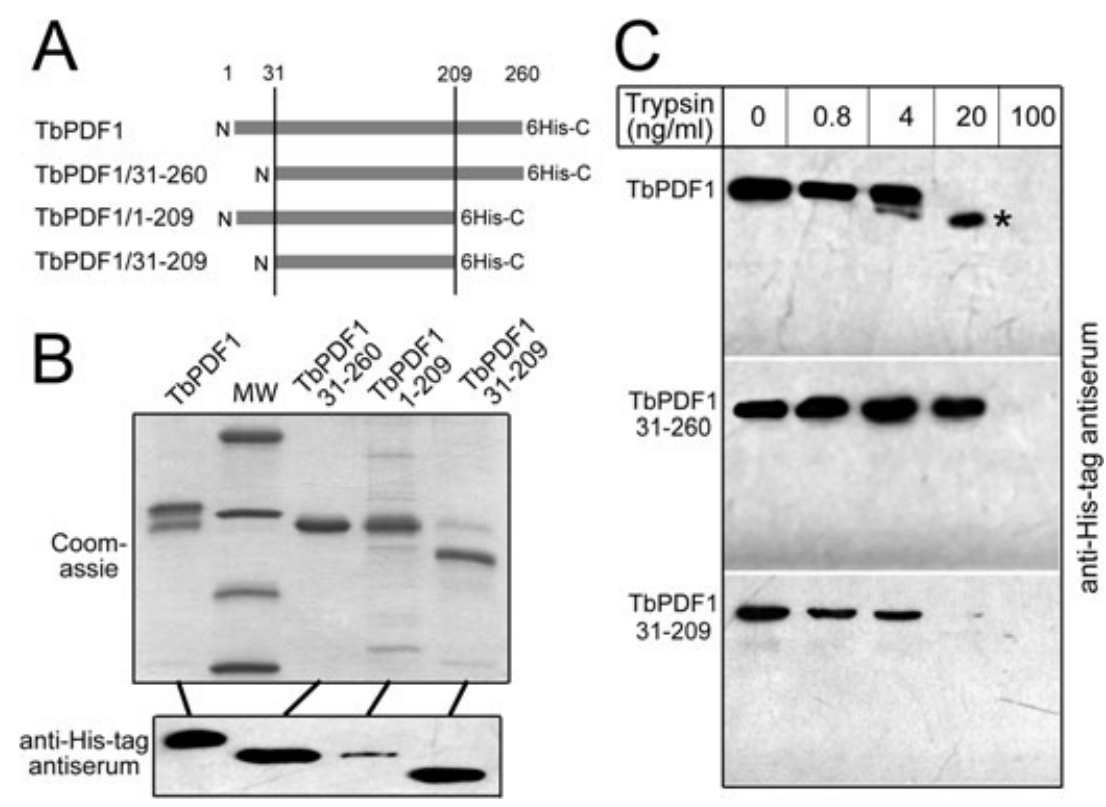

Fig. 2. Biochemical analysis of recombinant TbPDF1.

A. Schematic representation of the 6xHis-tagged TbPDF1 variants that were recombinantly expressed in E. coli.

B. Upper panel, Coomassie-stained $12 \%$ SDS polyacrylamide gel containing approximately $2 \mu \mathrm{g}$ each of Ni-NTA agarose-purified pool containing the recombinant proteins. In the case of TbPDF/1-209, purity was assessed to be less than $10 \%$ because of poor overexpression and co-elution with many contaminating proteins, including a major one co-eluting with most variants. The molecular weight markers in the second lane correspond to $43,30,20.1$ and $14.4 \mathrm{kDa}$. Lower panel, immunoblot analysis using anti-His-tag antibodies of a duplicate gel containing the same samples as in the upper panel.

C. Proteolytic digestion of the recombinant TbPDF1 variants with the indicated concentrations of trypsin $\left(\mathrm{ng} \mathrm{ml}^{-1}\right)$. Proteins were detected by immunoblot analysis using anti-His-tag antibodies. The asterisk shows the trypsin-resistant core of full-length TbPDF1 that in size corresponds to trypsin-resistant TbPDF1/31-260 seen in the middle panel.

enzyme unstable (Ragusa et al., 1998). Thus, this may also apply to TbPDF1, as measurements of the metal content show that zinc is present $\left(0.6 \pm 0.2\right.$ mole mole $^{-1}$ PDF) in all four variants of the protein. In analogy with results in E. coli (Meinnel and Blanquet, 1995), this indicates that zinc may have replaced another more efficient cation that is used in vivo (Ragusa et al., 1998). Unfortunately, as previously reported for human PDF (Lee et al., 2004), all attempts to optimize the activity by using oxidant scavengers, or by replacing zinc by other cations, were unsuccessful. Furthermore, we cannot exclude that imidazole elution from the nickel column could participate in enzyme inactivation via metal cation removal.

Finally, as the normally conserved glutamine in motif 1 of TbPDF1 is substituted by a lysine (Fig. 1), we have also expressed and purified a TbPDF1 variant where the conserved glutamine was reintroduced. However, the resulting TbPDF1 variant was not more active in deformylation than the wild-type one.

In summary, these results show that TbPDF1, similar to the PDF of humans and P. falciparum, has deformylase activity. Furthermore, as for other eukaryotic PDFs, the activity is not dependent on the presence or absence of the first $30 \mathrm{~N}$-terminal residues.

\section{Intracellular localization of TbPDF1 and TbPDF2}

Both trypanosomal PDF orthologues are predicted to have mitochondrial targeting signals. However, in T. brucei such predictions are difficult, as mitochondrial presequences can be very short (Häusler et al., 1997). Thus, in order to determine the localization of the two proteins, we prepared transgenic procyclic $T$. brucei cell lines, allowing inducible expression of TbPDF1 and TbPDF2 versions that carry the Ty1-peptide as a C-terminal epitope tag (Bastin et al., 1996). Immunoblot

Table 1. Substrate specificity of recombinant TbPDF1.

\begin{tabular}{lc}
\hline Peptide $^{\mathrm{a}}$ & Relative deformylation rate $^{\mathrm{b}}$ \\
\hline Fo-Met-X & 100 \\
Fo-Phe-X & 40 \\
Fo-Ala-X & 2 \\
Fo-lle-X & 3 \\
Fo-Met & $<<1$ \\
\hline
\end{tabular}

a. $\mathrm{X}$ indicates that the peptide is composed of an equimolar mixture of 19 usual amino acids.

b. Deformylation rates were measured using the TbPDF1/31-260. A value of 100 was given to the highest value. It corresponds to a deformylation rate of 13.5 pmole of hydrolysed peptide per minute and per mg protein added $\left(0.008 \mathrm{~s}^{-1}\right)$. Standard deviation was $<20 \%$. 
A
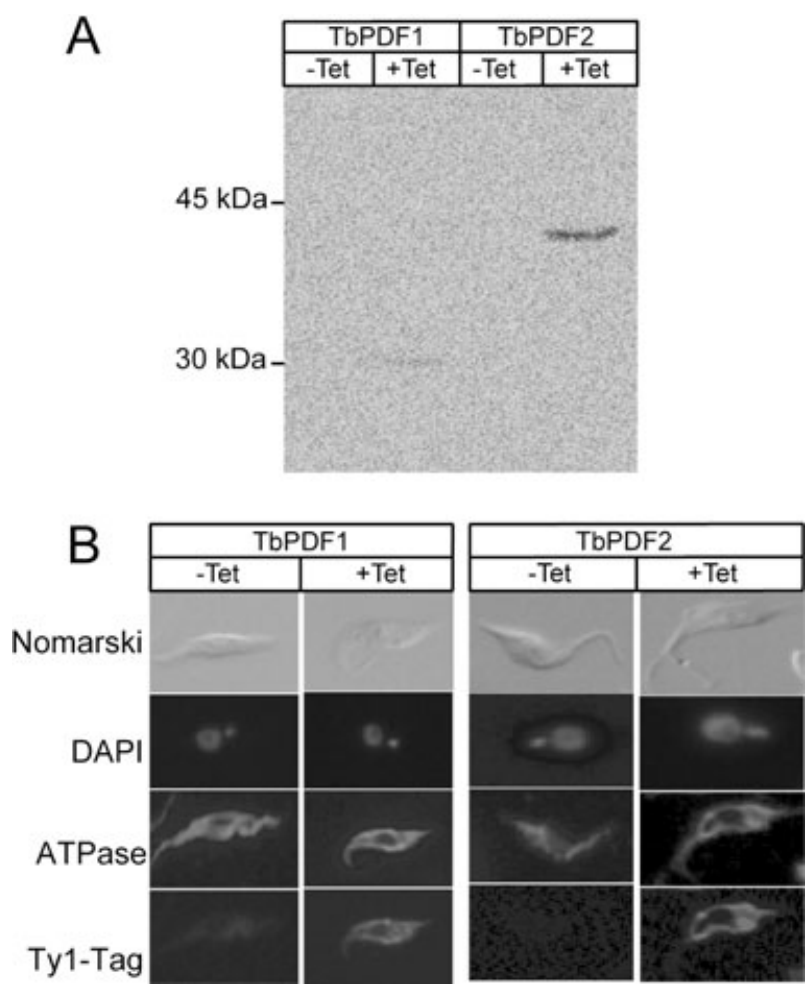

Fig. 3. Intracellular localization of TbPDF1 and TbPDF2.

Localization of trypanosomal PDF orthologues.

A. Immunoblot analysis of total cellular extracts of two transgenic T. brucei cell lines expressing TbPDF1 and TbPDF2 carrying Ty1-tags at their C-termini. Expression is under the control of the tetracycline inducible $(-,+$ Tet) procyclin promoter. The Ty1-tag was detected using the monoclonal BB2 antibody.

B. Immunofluoresence analysis of the same cell lines shown in A. The top two lines of each panels show the Nomarski picture and the DNA-staining using DAPI. The lower two lines of the panels depict the staining seen by using a polyclonal antibody against a subunit of the ATPase, which serves as a mitochondrial marker, and the staining obtained by using the Ty1-tag-specific monoclonal BB2 antibody.

analysis using anti-Ty1 antibody showed that, in both induced cell lines, a single band was detected that corresponded to the expected molecular weights of TbPDF1 and TbPDF2 respectively (Fig. 3A). Immunofluorescence of either of the two cell lines revealed a reticulate staining pattern, specific for induced cells, that is identical to the one seen for the mitochondrial marker (Fig. 3B). Thus, these results show that both TbPDF1 and TbPDF2 are localized in mitochondria.

\section{Effect of RNAi-mediated ablation of TbPDF1 and TbPDF2 on growth}

In order to study the importance of TbPDF1 and TbPDF2 for growth and survival of procyclic $T$. brucei, we established transgenic cell lines that allow individual or combined RNAi-mediated ablation of the two enzymes. Growth of the RNAi cell lines was tested (i) in the standard medium SDM-79 (Brun and Schönenberger, 1979), which contains proline and glucose as the major energy sources (Fig. 4A and B), as well as (ii) in SDM-80 (Lamour et al., 2005), a modified version of SDM-79 that lacks glucose (Fig. 4C).

A
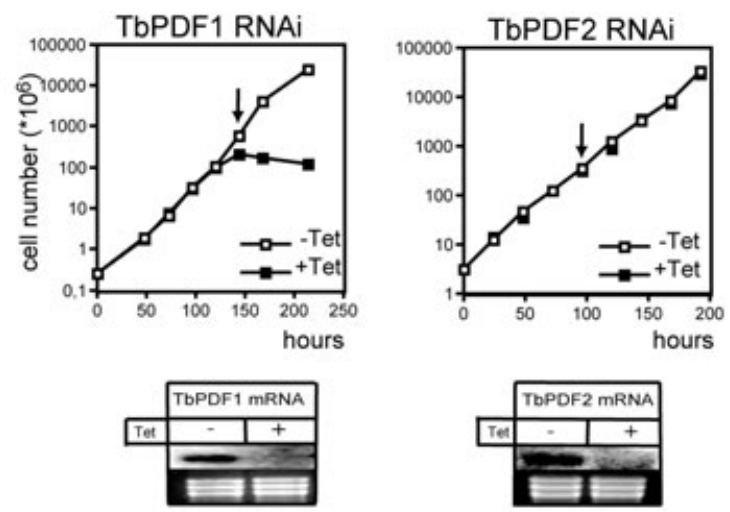

B
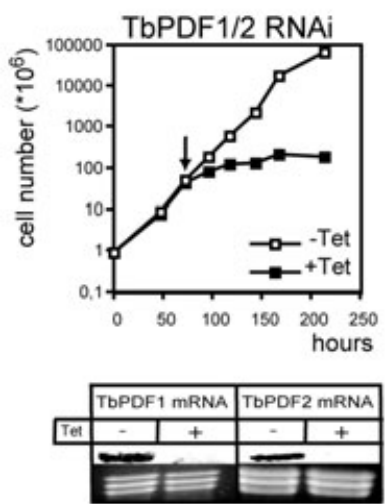

C
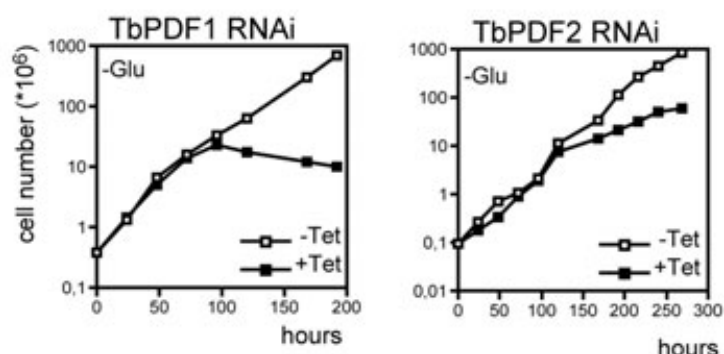

Fig. 4. Effect of RNAi-mediated ablation of TbPDF1 and TbPDF2 on growth.

A. Growth curves in standard culture medium SDM-79 of uninduced and induced ( - , +Tet) clonal TbPDF1 (left panel) and TbPDF2 (right panel) RNAi cell lines. The panels below the graphs show Northern blots for the corresponding TbPDF mRNAs. The rRNAs in the lower panel serve as loading controls. Time of sampling is indicated by the arrows.

B. Growth curve in standard culture medium SDM-79 of an uninduced and induced $(-,+$ Tet) culture of a representative clonal TbPDF1/TbPDF2 double-RNAi cell line. The panel below the graph shows a Northern blot for the two TbPDF mRNAs.

C. Growth curves of uninduced and induced TbPDF1 and TbPDF2 RNAi cell lines in culture medium SDM-80 that lacks glucose (- Glu). All growth curves have been determined at least three times in independent experiments and were shown to be highly reproducible. 
Ablation of TbPDF1 caused a growth arrest in SDM-79 after approximately $150 \mathrm{~h}$ of induction (Fig. 4A, left graph). However, when cells ablated for TbPDF2 were tested in the same medium, growth was not affected. Thus, TbPDF2, in contrast to TbPDF1, appears to be dispensable for growth on SDM-79. However, a doubleRNAi cell line allowing simultaneous ablation of TbPDF1 and TbPDF2 (Fig. 4B) shows a growth arrest after approximately $70 \mathrm{~h}$ of induction, much earlier than the $150 \mathrm{~h}$ that is needed if only TbPDF1 is ablated. These results indicate that, whereas a reduced level of TbPDF2 alone does not affect growth in SDM-79, it causes the growth arrest induced by ablation of TbPDF1 to occur much earlier.

When tested in glucose-free SDM-80 medium, the growth arrest caused by ablation of TbPDF1 occurs already approximately $90 \mathrm{~h}$ after induction (Fig. 4C, left panel). Furthermore, in SDM-80, unlike in SDM-79, a slow growth phenotype is also detected for the induced TbPDF2 RNAi cell line (Fig. 4C, right panel).

In procyclic T. brucei, glucose, after conversion into pyruvate, is mainly used for mitochondrial substrate-level phosphorylation (SUBPHOS) in the trypanosome-specific acetate:succinate CoA transferase/succinyl-CoA synthetase (ASCT) cycle (Van Hellemond et al., 1998) and, not as might be expected, fed into the citric acid cycle (Bochud-Allemann and Schneider, 2002; van Weelden et al., 2003). When grown in SDM-79, where glucose is available, the energy needs of $T$. brucei can be fulfilled mainly by SUBPHOS. In the glucose-free SDM-80 medium, however, the sole energy source is proline that can only be utilized by OXPHOS. Growth in SDM-80 therefore selects for cells capable of performing efficient OXPHOS (Lamour et al., 2005). Thus, the fact that ablation of either of the two proteins causes a stronger growth phenotype in glucose-free than in the standard medium suggests that TbPDF1 and to a more limited extent also TbPDF2 are, at least indirectly, required for efficient OXPHOS.

\section{Effect of RNAi-mediated ablation of TbPDF1 and TbPDF2 on mitochondrial ATP production}

The proteins encoded in the mitochondrion of T. bruceithe substrates of TbPDF1 and possibly TbPDF2 - either function for the most part directly in OXPHOS, or are components of the mitochondrial translation machinery that produces them (Feagin, 2000). Thus, should removal of the formyl group be essential for the function of any of these mitochondrially encoded proteins, the lack of PDF activity should ultimately affect OXPHOS. The mitochondrion of procyclic T. brucei produces ATP, by OXPHOS as well as by SUBPHOS linked to the citric acid cycle or the ASCT cycle (Besteiro et al., 2005). Using isolated mito- chondria, we have recently established an assay that allows quantification of both modes of ATP production (Bochud-Allemann and Schneider, 2002; Schneider et al., 2007). In these assays, OXPHOS is induced by the addition of either succinate or glycerol-3 phosphate. As expected, this type of ATP production is antimycin sensitive. Mitochondrial SUBPHOS, which is resistant to antimycin, is induced by the addition of $\alpha$-ketoglutarate (for the SUBPHOS in the citric acid cycle) or pyruvate (for the SUBPHOS in the ASCT cycle). In the latter case, succinate has to be added as a co-substrate. Thus, $25-50 \%$ of the ATP produced by the combination of pyruvate and succinate is due to OXPHOS and therefore antimycin sensitive (Bochud-Allemann and Schneider, 2002). Isolated mitochondria of $T$. brucei are depleted for nucleotides; therefore, ADP has to be added in all assays. Atractyloside treatment prevents mitochondrial import of the added ADP and serves as a control for all modes of mitochondrial ATP production. Figure 5A shows that, in line with the deformylase activity of the recombinant protein, ablation of TbPDF1 results in a loss of OXPHOS induced either by glycerol-3 phosphate or by succinate, but does not affect mitochondrial SUBPHOS assayed by pyruvate or $\alpha$-ketoglutarate respectively. Ablation of TbPDF2, on the other hand, does not influence either mode of ATP production (Fig. 5B). Thus, TbPDF1, but not TbPDF2, is required for OXPHOS in isolated organelles. These results are in agreement with the observation that TbPDF1 is required for growth in any medium, and explain why the growth arrest during TbPDF1 RNAi appears earlier in glucose-free than in the standard medium (Fig. 4). Isolated mitochondria ablated for TbPDF2 are not impaired in OXPHOS, which is consistent with the normal growth of the induced RNAi cell line that is observed in standard medium. However, ablation of TbPDF2 reinforces the growth arrest of cells that are already ablated for TbPDF1 and leads to a slow growth phenotype in glucose-free medium (Fig. 4). This suggests that while TbPDF2 is not required for the level of OXPHOS that is needed under normal growth conditions, it is nevertheless required for the highly efficient OXPHOS that is demanded in glucose-free medium.

\section{Discussion}

A phylogenetic analysis based on alignments of the three conserved motifs suggests that the trypanosomal PDFs are most closely related to the PDFs of Archaea (Giglione and Meinnel, 2001b). In fact, archaeal and trypanosomatidal PDFs are sufficiently different from all other PDFs to classify them in an own group, termed type 3 PDFs (Giglione et al., 2004). Archaea do not use formyl-methionine to initiate protein synthesis; thus, it is not known which reaction archaeal PDF orthologues catalyse. Interestingly, 
A

TbPDF1 RNAi

$\frac{}{0}$
0
$\frac{0}{\alpha}$
0
0
0
0
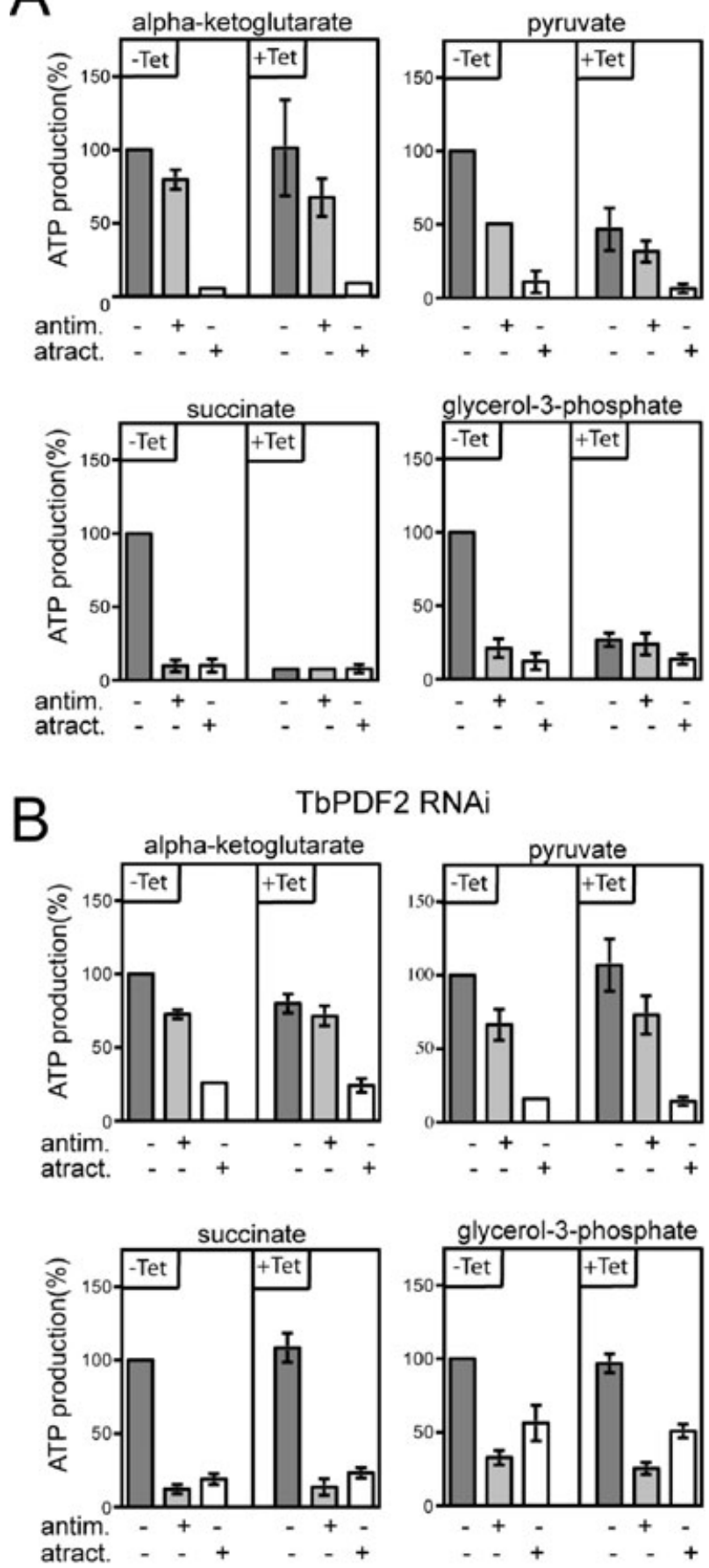

Fig. 5. Effect of RNAi-mediated ablation of TbPDF1 and TbPDF2 on mitochondrial ATP production. OXPHOS, induced by succinate or glycerol-3 phosphate, and SUBPHOS, induced by $\alpha$-ketoglutarate or pyruvate, were assayed in crude mitochondrial fractions of TbPDF1 (A) and TbPDF2 (B) RNAi cell lines respectively. Uninduced cells (- Tet) are shown on the left, induced cells (+ Tet) on the right of each panel. The substrate tested is indicated at the top and additions of antimycine (antim.) and atractyloside (atract.) at the bottom of each panel. ATP productions in mitochondria isolated from uninduced cells tested without antimycine or atractyloside are set to $100 \%$. The bars represent means expressed as percentages from three or more independent inductions. Standard errors are indicated. however, recombinant PDF1 of T. brucei, a type 3 enzyme, exhibits deformylase activity whose specificity mirrors that of bacterial enzymes. The specific activity of the recombinant protein is low but comparable to that of human and $P$. falciparum PDFs that belong to class $1 \mathrm{~A}$ and $1 \mathrm{~B}$ respectively (Giglione et al., 2004). Other features that are shared between TbPDF1 and the wellcharacterized bacterial PDFs are a trypsin-resistant active core of similar size and the capability to bind metals. In fact, it is likely that an exchange of a metal ion, of yet unknown nature, for zinc in the recombinant enzyme is responsible for the low specific activity that is observed.

We have previously established that active MTF is present in the mitochondrion of T. brucei (Tan et al., 2002). Unexpectedly, due to the absence of mitochondrial tRNA genes in T. brucei, the tRNA that becomes formylated is the elongator tRNA ${ }^{\mathrm{Met}}$ imported from the cytosol. The proteins encoded on the T. brucei mitochondrial DNA are subunits I-III of cytochrome oxidase, cytochrome b, subunit 6 of the ATPase, the ribosomal protein Rps12, and at least seven subunits of the NADH dehydrogenase (Feagin, 2000). When translated, the corresponding nascent proteins will necessarily carry a formyl-methionine at their $\mathrm{N}$-termini, because of mitochondrial MTF activity. Thus, the only compartment in T. brucei where substrates for PDFs are present is the mitochondrion. In agreement with this, TbPDF1 is localized to mitochondria. However, up to now the N-termini of only two mitochondrially synthesized proteins, cytochrome $b$ and cytochrome oxidase subunit I (Horvath et al., 2000a, b), have directly been sequenced. Both of these proteins had to be treated with $\mathrm{HCl}$ prior to sequencing because their $\mathrm{N}$-termini were blocked, suggesting that they consisted of an unprocessed formylmethionine. Thus, in vivo evidence for deformylation of mitochondrially encoded proteins is still lacking, as is also the case for human mitochondrial proteins (Serero et al., 2003).

Isolated mitochondria from TbPDF1-ablated cells show a specific loss of OXPHOS, but not of SUBPHOS. In agreement with this, the growth arrest induced by ablation of TbPDF1 occurs much earlier in glucose-free medium, that selects for efficient OXPHOS, than in the standard medium that provides substrates for OXPHOS and mitochondrial SUBPHOS. The substrates of PDFs are mitochondrially encoded proteins, many of which are required for OXPHOS but not for mitochondrial SUBPHOS. These results therefore support the idea that TbPDF1 is a deformylase.

Unexpectedly, unlike humans (Nguyen et al., 2003; Serero et al., 2003; Lee et al., 2004) but similar to plants (Giglione et al., 2000), T. brucei has two PDF homologues, both of which are localized in mitochondria. In contrast to TbPDF1, TbPDF2 is dispensable for growth on standard medium. Interestingly, however, TbPDF2- 
ablated cells show a slow growth phenotype on glucosefree medium, suggesting that the function of TbPDF2 is also, at least indirectly, linked to OXPHOS. Furthermore, the synergistic growth phenotype observed in the cell line ablated for both trypanosomal PDFs suggests that the two proteins are functionally connected.

Given the strong homology of the catalytic domain of TbPDF2 with other well-characterized PDFs (Giglione and Meinnel, 2001b), it appears likely that it also has deformylase activity. Thus, TbPDF1 and TbPDF2 may in part have overlapping functions: TbPDF1 may deformylate all mitochondrially synthesized proteins, although some only inefficiently, whereas TbPDF2 might be more selective and only deformylate proteins that are poorly recognized by TbPDF1. However, attempts to purify recombinant TbPDF2 have so far been unsuccessful. Furthermore, overexpression of TbPDF2 did not rescue the growth arrest that is observed in the induced TbPDF1 RNAi cell line. The described scenario is therefore mainly based on speculation, and the function of TbPDF2 remains unknown at present.

Why are there two PDF orthologues in T. brucei? Interestingly, plants also have two of these enzymes (Giglione et al., 2000). One is essentially localized in mitochondria (PDF1A), whereas the other (PDF1B) is found in both plastids and mitochondria. Unlike PDF1A, PDF1B is essential for chloroplast function (Giglione and Meinnel, 2001a). Nevertheless, the two proteins are in part functionally redundant. Hence, overexpression of PDF1A partially compensates for the absence of PDF1B in chloroplasts, and overexpression of PDF1A can fully complement the lack of PDF1B (Giglione et al., 2003). Plant PDFs are believed to mainly differ by their capacity to bind distinct metal cation, $\mathrm{Zn}^{2+}$ for PDF1A and $\mathrm{Fe}^{2+}$ for PDF1B (Serero et al., 2001). Consequently, a unique feature of PDF1B is its strong sensitivity to oxidation. This led to the proposal that PDF1A was suited for environments prone to oxidative stress such as mitochondria (Serero et al., 2001). Trypanosomatids are closely related to the euglenids, most of which have a plastid. Furthermore, a number of plant-like genes encoding homologues of proteins found in chloroplasts have been found in the genome of T. brucei (Hannaert et al. 2003). Thus, it has been suggested that $T$. brucei may once have had a plastid which it has lost secondarily (Martin and Borst, 2003). It is therefore likely that one of the two trypanosomal PDFs represents an evolutionary remnant of a PDF that originally was also targeted to the plastid. However, even if the evolutionary history of one of the trypanosomal PDFs is linked to plastids, it is clear that today both proteins have a mitochondrial function. TbPDF1 is strictly required for OXPHOS under all conditions. The requirement of TbPDF2 is less stringent but is seen when growth is tested in glucose-free medium or at limiting concentra- tions of TbPDF1. Finally, like in plants, it is not unlikely that the nature of the metal cation bound in vivo could be different between the two trypanosomal PDFs, each being more adapted to unique environmental stress conditions.

Recently, a PDF orthologue has been identified and characterized in P. falciparum (Meinnel, 2000; BracchiRicard et al., 2001). PDF inhibitors were shown to interfere with growth of the intraerythrocytic stage of the parasite (Bracchi-Ricard et al., 2001; Wiesner et al., 2001). Based on these observations, it has been proposed that the plasmodial PDF could be exploited as a novel target for antimalarial drugs (Meinnel, 2000; Giglione and Meinnel, 2001b). The PDF orthologues of $T$. brucei are essential for mitochondrial function, and their sequence is quite divergent from other eukaryotic PDFs. This suggests that also the trypanosomal PDFs may be suitable drug targets. Thus, it is possible that the trypanosomal PDFs are required for processing of subunit 6 of the ATPase, a mitochondrially encoded protein that was demonstrated to be essential for both life-cycle stages (Schnaufer et al., 2005). Unlike T. brucei, for which OXPHOS is specific for the insect stage and does not occur in the bloodstream form of the parasite (Durieux et al., 1991; Schneider, 2001), Leishmania and T. cruzi need a functional respiratory chain for survival in their respective vertebrate hosts (Van Hellemond and Tielens, 1997). Thus, in T. cruzi and Leishmania, PDF orthologues appear to be excellent drug targets to fight the associated diseases.

\section{Experimental procedures}

\section{Purification of recombinant TbPDF1 from E. coli}

The ORFs corresponding to full-length TbPDF1 (Fig. 1) and the described variants thereof (Fig. 2) were cloned into pQE60 (Qiagen), a vector specialized for in-frame fusions with a C-terminal 6xHis tag. Transfected E. coli M15 was grown at $30^{\circ} \mathrm{C}$ in $2 x T Y$ containing $50 \mu \mathrm{g} \mathrm{m}^{-1}$ ampicillin and $25 \mu \mathrm{g} \mathrm{ml}^{-1}$ kanamycin. Expression of the recombinant proteins was induced with $0.1 \mathrm{mM} \mathrm{IPTG}$ at an $\mathrm{OD}_{650}$ of 0.9 for $4 \mathrm{~h}$. Cells were harvested and resuspended in $20 \mathrm{mM}$ Tris$\mathrm{HCl}$ pH 8.0, 20 mM 2-mercaptoethanol, 0.3 M NaCl (buffer A) containing $10 \mathrm{mM}$ imidazole. The sample was sonicated, and cell debris removed by centrifugation. TbPDF1 recombinant proteins were purified by affinity chromatography on Ni-NTA Agarose columns (Qiagen). The columns were washed with an excess of buffer A containing $20 \mathrm{mM}$ imidazole, and the His-tagged proteins were eluted with a linear $20-110 \mathrm{mM}$ imidazole gradient in buffer $\mathrm{A}$. The eluted proteins were dialysed against $20 \mathrm{mM}$ Tris ( $\mathrm{pH} 8.0$ ), $1 \mathrm{mM}$ 2-mercaptoethanol containing $55 \%$ glycerol and stored in aliquots at $-30^{\circ} \mathrm{C}$. Purity was assessed to be $70 \%$ (TbPDF1), 95\% (TbPDF1/ 31-260), 10\% (TbPDF1/1-209) and 60\% (TbPDF1/31-209).

\section{Biochemistry}

Peptide deformylase (PDF) activity assays were carried out in $50 \mathrm{mM}$ HEPES ( $\mathrm{pH} \mathrm{7.0)}$ at $37^{\circ} \mathrm{C}$ for $90 \mathrm{~min}$ as described 
(Meinnel and Blanquet, 1995). Proteolytic digestion with different concentrations of trypsin (Sigma) of the indicated PDF variants (Fig. 2) was performed in $0.1 \mathrm{M}$ Tris- $\mathrm{HCl}(\mathrm{pH} 7.7)$ containing $1 \mathrm{mM}$ 2-mercaptoethanol. Samples were incubated at $37^{\circ} \mathrm{C}$ for $15 \mathrm{~min}$, and the reaction was stopped by the addition of chicken egg-white ovomucoid 2/1 (w/w) with respect to trypsin. Finally, the reaction products were analysed by $12 \%$ SDS-PAGE. To measure zinc content by atomic absorption spectroscopy, protein samples were dialysed overnight against a buffer containing $20 \mathrm{mM}$ Tris- $\mathrm{HCl}$ $(\mathrm{pH} 7.5)$ containing $0.1 \mathrm{M} \mathrm{KCl}$. Atomic absorbencies were measured at $213.9 \mathrm{~nm}$ in the peak height mode during $5 \mathrm{~s}$ after $0.1 \mathrm{ml}$ injections using a Varian AA220 spectrophotometer equipped with an air-acetylene burner (Serero et al., 2003). Zinc concentrations in serial dilutions of the enzyme samples were calculated by comparison with standard solutions of known concentrations.

\section{Production of transgenic T. brucei cell lines}

As a tag to localize TbPDF1 and TbPDF2, a 10-amino-acid epitope of the major structural protein of the yeast Ty1 viruslike particle was used which is recognized by the monoclonal antibody BB2 (Bastin et al., 1996). The sequences corresponding to the $\mathrm{C}$-terminal Ty1-tagged proteins were cloned into a derivative of the plasmid pLew-100, which allows tetracycline inducible expression of the tagged proteins (Wirtz et al., 1999). RNAi of TbPDF1 and TbPDF2 was performed using stem-loop constructs containing the phleomycinresistance gene as described (Bochud-Allemann and Schneider, 2002). As inserts we used a 715 bp fragment (nucleotides 65-779) of the TbPDF1 gene and a $644 \mathrm{bp}$ fragment of the TbPDF2 gene (nucleotides 2-645). To obtain the double-RNAi cell lines analysed in Fig. 4B, we replaced the phleomycine-resistance gene of the construct used to ablate TbPDF2 with the puromycine-resistance gene, and used the resulting plasmid to transfect the previously characterized single knock-down for TbPDF1. Transfection of T. brucei, selection with antibiotics, cloning and induction with tetracycline were conducted as described (McCulloch et al., 2004). All transgenic cell lines used in this study are based on T. brucei strain 29-13, which was grown in SDM-79 (Brun and Schönenberger, 1979) supplemented with 15\% FCS, $50 \mu \mathrm{g} \mathrm{ml}^{-1}$ hygromycin and $15 \mu \mathrm{g} \mathrm{ml}^{-1} \mathrm{G}-418$. Growth of RNAi cells was also analysed in SDM-80, a modified SDM-79 that lacks glucose and which is supplemented with $10 \%$ of dialysed FCS as described (Lamour et al., 2005).

\section{In organello ATP production assays}

ATP production assays were performed as described (Schneider et al., 2007). In total, $10^{7}$ cell equivalents of the digitonin-extracted cells from the TbPDF1 RNAi cell line and the TbPDF2 RNAi cell line were used for each assay. Induction times for the TbPDF1 and TbPDF2 RNAi cell lines used for the experiments shown in Fig. 5 were 160 and $100 \mathrm{~h}$ respectively. However, for the TbPDF2 RNAi cell lines, the same results were obtained for longer induction times. To induce ATP production, $5 \mathrm{mM}$ of the indicated substrates (succinate, glycerol-3 phosphate, $\alpha$-ketoglutarate or pyruvate/succinate) and $67 \mu \mathrm{M}$ of ADP was added. After incubation at $27^{\circ} \mathrm{C}$ for $30 \mathrm{~min}$, the reaction was processed and the ATP concentration determined as described. Inhibitors were preincubated with mitochondria for $10 \mathrm{~min}$ on ice and used at the following final concentrations: atractyloside $\left(33.3 \mu \mathrm{g} \mathrm{ml}^{-1}\right)$ and antimycin $(2.7 \mu \mathrm{M})$. ATP production was then induced by the addition of substrate and ADP.

\section{Miscellaneous}

RNA for Northern analysis was isolated by the acid guanidinium method (Chomczyinski and Sacchi, 1987). The probes used were random hexamer $\alpha^{-32} \mathrm{P}$ dCTP-labelled double-stranded DNA fragments corresponding to nucleotides $65-779$ of the TbPDF1 gene and to nucleotides 2-645 of the TbPDF2 gene respectively. Immunofluorescence was performed as described (Schneider et al., 1987).

\section{Acknowledgements}

We thank $\mathrm{Dr}$ G. Cross for providing us with the pLew100 vector and the T. brucei 29-13 strain. We also thank Dr Christine Lazennec for efficient technical assistance at the initial stage of the project. Dr Elisabetta Ullu and Sara Melville are acknowledged for help in identifying and making available the bacterial stabs of the T. brucei cosmid collection. This study was supported by Grants 3100A0-109311 from the Swiss National Foundation.

\section{References}

Bastin, P., Bagherzadeh, A., Matthews, K.R., and Gull, K. (1996) A novel epitope tag system to study protein targeting and organelle biogenesis in Trypanosoma brucei. Mol Biochem Parasitol 77: 235-239.

Besteiro, S., Barrett, M.P., Riviere, L., and Bringaud, F. (2005) Energy generation in insect stages of Trypanosoma brucei: metabolism in flux. Trends Parasitol 21: 185-191.

Bhattacharyya, S.N., and Adhya, S. (2004) The complexity of mitochondrial tRNA import. RNA Biology 1: 84-88.

Bochud-Allemann, N., and Schneider, A. (2002) Mitochondrial substrate level phosphorylation is essential for growth of procyclic Trypanosoma brucei. J Biol Chem 277: 3284932854.

Boularot, A., Giglione, C., Artaud, I., and Meinnel, T. (2004) Structure-activity relationship analysis and therapeutic potential of peptide deformylase inhibitors. Curr Opin Investig Drugs 5: 809-822.

Bracchi-Ricard, V., Nguyen, K.T., Zhou, Y., Rajagopalan, P.T.R., Chakrabarti, D., and Pei, D. (2001) Characterization of an eukaryotic peptide deformylase from Plasmodium falciparum. Arch Biochem Biophys 396: 162-170.

Brun, R., and Schönenberger, M. (1979) Cultivation an in vitro cloning of procyclic culture forms of Trypansoma brucei in a semi-defined medium. Acta Tropica 36: 289292.

Charrière, F., Tan, T.H.P., and Schneider, A. (2005) Mitochondrial initiation factor 2 of Trypanosoma brucei binds imported formylated elongator-type methionyl-tRNA. J Biol Chem 280: 15659-15665. 
Chomczyinski, P., and Sacchi, N. (1987) Single-step method of RNA isolation by acid guanidinium thiocyanate-phenolchloroform extraction. Anal Biochem 162: 156-159.

Durieux, P.O., Schutz, P., Brun, R., and Kohler, P. (1991) Alterations in Krebs cycle enzyme activities and carbohydrate catabolism in two strains of Trypanosoma brucei during in vitro differentiation of their bloodstream to procyclic stages. Mol Biochem Parasitol 45: 19-27.

Feagin, J.E. (2000) Mitochondrial genome diversity in parasites. Int J Parasitol 30: 371-390.

Giglione, C., and Meinnel, T. (2001a) Organellar peptide deformylases: universality of the $\mathrm{N}$-terminal methionine cleavage mechanism. Trends Plant Sci 6: 566-572.

Giglione, C., and Meinnel, T. (2001b) Peptide deformylase as an emerging target for antiparasitic agents. Emerging Therapeutic Targets 5: 41-57.

Giglione, C., Serero, A., Pierre, M., Boisson, B., and Meinnel, T. (2000) Identification of eukaryotic peptide deformylases reveals universality of $\mathrm{N}$-terminal protein processing mechanisms. EMBO J 19: 5916-5929.

Giglione, C., Vallon, O., and Meinnel, T. (2003) Control of protein life-span by $\mathrm{N}$-terminal methionine excision. EMBO J 22: 13-23.

Giglione, C., Boularot, A., and Meinnel, T. (2004) Protein N-terminal methionine excision. Cell Mol Life Sci61: 14551474.

Hannaert, V., Saavedra, E., Duffieux, F., Szikora, J.P., Rigden, D.J., Michels, P.A., and Opperdoes, F.R. (2003) Plant-like traits associated with metabolism of Trypanosoma parasites. Proc Natl Acad Sci USA 100: 1067-1071.

Häusler, T., Stierhof, Y.-D., Blattner, J., and Clayton, C. (1997) Conservation of mitochondrial targeting sequence function in mitochondrial and hydrogenosomal proteins from the early-branching eukaryotes Crithidia, Trypanosoma and Trichomonas. Eur J Cell Biol 73: 240-251.

van Hellemond, J.J., and Tielens, A.G.M. (1997) Inhibition of the respiratory chain results on a reversible metabolic arrest in Leishmania promastigotes. Mol Biochem Parasitol 85: $135-138$.

van Hellemond, J.J., Opperdoes, F.R., and Tielens, A.G.M. (1998) Trypanosomatides produce acetate via a mitochondrial acetate: succinate CoA transferase. Proc Natl Acad Sci USA 95: 3036-3041.

Horvath, A., Berry, E.A., and Maslov, D.A. (2000a) Translation of the edited mRNA for cytochrome $b$ in trypanosome mitochondria. Science 287: 1639-1640.

Horvath, A., Kingan, T.G., and Maslov, D.A. (2000b) Detection of the mitochondrially encoded cytochrome $c$ oxidase subunit $I$ in the trypanosomatid protozoan Leishmania tarentolae. J Biol Chem 275: 17160-17165.

Lamour, N., Riviere, L., Coustou, V., Coombs, G.H., Barrett, M.P., and Bringaud, F. (2005) Proline metabolism in procyclic Trypanosoma brucei is down-regulated in the presence of glucose. J Biol Chem 280: 11902-11910.

Lee, M.D., She, Y., Soskis, M.J., Borella, C.P., Gardner, J.R., Hayes, P.A., et al. (2004) Human mitochondrial peptide deformylase, a new anticancer target of actinonin-based antibiotics. J Clin Invest 114: 1107-1116.

Leeds, J.A., and Dean, C.R. (2006) Peptide deformylase as an antibacterial target: a critical assessment. Curr Opin Pharmacol 5: 445-452.
Lüscher, A., de Koning, H., and Mäser, P. (2007) Chemotherapeutic strategies against Trypanosoma brucei: drug targets vs. drug targeting. Curr Pharm Des 13: 555-567.

McCulloch, R., Vassella, E., Burton, P., Boshart, M., and Barry, J.D. (2004) Transformation of monomorphic and pleomorphic Trypanosoma brucei. Methods Mol Biol 262: 53-86.

Martin, N.C. (2002) Location alters tRNA identity: Trypanosoma bruceis cytosolic elongator methionyl tRNA is both the initiator and elongator in mitochondria. Proc Natl Acad Sci USA 99: 1110-1112.

Martin, W., and Borst, P. (2003) Secondary loss of chlorosplasts in trypanosomes. Proc Natl Acad Sci USA 100: 765-767.

Mayer, C., Stortchevoi, A., Kohrer, C., Varshney, U., and RajBhandary, U.L. (2001) Initiator tRNA and its role in initiation of protein synthesis. Cold Spring Harb Symp Quant Biol 66: 195-206.

Meinnel, T. (2000) Peptide deformylase of eukaryotic: a target for new antiparasitic agents? Parasitol Today 16: 165-168.

Meinnel, T., and Blanquet, S. (1995) Enzymatic properties of Escherichia coli peptide deformylase. J Bact 177: 18831887.

Meinnel, T., Lazennec, C., Dardel, F., Schmitter, J.M., and Blanquet, S. (1996) The C-terminal domain of peptide deformylase is disordered and dispensable for activity. FEBS Lett 385: 91-95.

Meinnel, T., Lazennec, C., Villoing, S., and Blanquet, S. (1997) Structure-function relationships within the peptide deformylase family. Evidence for a conserved architecture of the active site involving three conserved motifs and a metal ion. J Mol Biol 267: 749-761.

Nguyen, K.T., Hu, X., Colton, C., Chakrabarti, R., Zhu, M.X., and Pei, D. (2003) Characterization of a human peptide deformylase: implications for antibacterial drug design. Biochemistry 42: 9952-9958.

Ragusa, S., Blanquet, S., and Meinnel, T. (1998) Control of peptide deformylase activity by metal cations. $J \mathrm{Mol}$ Biol 280: 515-523.

Ragusa, S., Mouchet, P., Lazennec, C., Dive, V., and Meinnel, T. (1999) Substrate recognition and selectivity of peptide deformylase. Similarities and differences with metzincins and thermolysin. $J$ Mol Biol 289: 1445-1457.

Schnaufer, A., Clark-Walker, G.D., Steinberg, A.G., and Stuart, K. (2005) The F1-ATP synthase complex in bloodstream stage trypanosomes has an unusual and essential function. EMBO J 24: 4029-4040.

Schneider, A. (2001) Unique aspects of mitochondrial biogenesis in trypanosomatids. Int J Parasito/ 31: 1403-1415.

Schneider, A., and Marechal-Drouard, L. (2000) Mitochondrial tRNA import: are there distinct mechanisms? Trends Cell Biol 10: 509-513.

Schneider, A., Sherwin, T., Sasse, R., Russell, D., Gull, K., and Seebeck, T. (1987) Subpellicular and flagellar microtubules of Trypanosoma brucei contain the same alpha tubulin isotypes. J Cell Biol 104: 431-438.

Schneider, A., Bouzaidi-Tiali, N., Chanez, A.-L., and Bulliard, L. (2007) ATP production in isolated mitochondria of procyclic Trypanosoma brucei. Methods Mol Biol 372: 379387. 
Serero, A., Giglione, C., and Meinnel, T. (2001) Distinctive features of the two classes of eukaryotic peptide deformylases. J Mol Biol 314: 695-708.

Serero, A., Giglione, C., Sardini, A., Martinez-Sanz, J., and Meinnel, T. (2003) An unusual peptide deformylase features in the human mitochondrial N-terminal methionine excision pathway. J Biol Chem 278: 52953-52963.

Tan, T.H.P., Bochud-Allemannn, N., Horn, E.K., and Schneider, A. (2002) Eukaryotic-type elongator tRNAMet of Trypanosoma brucei becomes formylated after import into mitochondria. Proc Natl Acad Sci USA 99: 1152-1157. van Weelden, S.W., Fast, B., Vogt, A., van der Meer, P., Saas, J., van Hellemond, J.J., et al. (2003) Procyclic Trypanosoma brucei do not use Krebs cycle activity for energy generation. J Biol Chem 278: 12854-12863.

Wiesner, J., Sanderbrand, S., Altincicek, B., Beck, E., and Jomaa, H. (2001) Seeking new targets for antiparasitic agents. Trends Parasitol 17: 7-8.

Wirtz, E., Leal, S., Ochatt, C., and Cross, G.A. (1999) A tightly regulated inducible expression system for conditional gene knock-outs and dominant-negative genetics in Trypanosoma brucei. Mol Biochem Parasitol 99: 89-101. 